\title{
El trabajo en la contabilidad pública y las percepciones de la Generación Y
}

\author{
Marisela Santiago Castro \\ Universidad de Puerto Rico, Río Piedras
}

Recibido: 20 de septiembre de 2013

Aceptado: 9 de diciembre de 2013

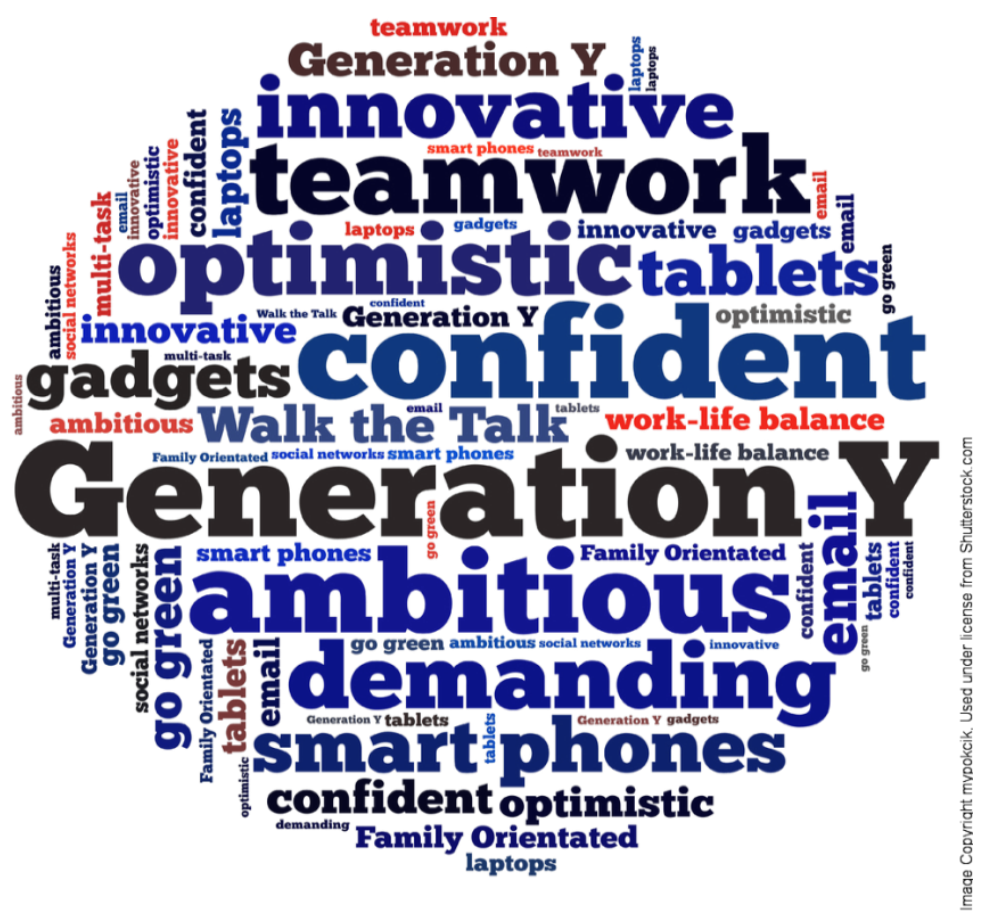

\section{RESUMEN}

El artículo presenta los resultados de una encuesta a una muestra de estudiantes de La Generación Y sobre las percepciones del trabajo en contabilidad pública. Se encontró que la mayoría de los encuestados aspiran a continuar estudios graduados una vez finalicen su grado subgraduado, no necesariamente a solo obtener la licencia de contador público autorizado (CPA). Además que los factores que atraen a los estudiantes a decidir por la contabilidad pública como carrera son: la oportunidad de trabajar con diferentes clientes y obtener una mejor experiencia e incentivos para las certificaciones profesionales. Los resultados de esta investigación pueden ser de interés para las personas que llevan a cabo tareas de reclutamiento para las firmas de contabilidad porque aporta información adicional sobre las necesidades y percepciones de sus posibles futuros empleados. También pueden ser aplicados a otras áreas de los negocios.

PALABRAS CLAVES: Contabilidad pública, Generación Y, carrera profesional, percepciones

\begin{abstract}
The article presents the results a survey to a sample of students from Generation Y about their perceptions of the work in public accounting. The majority of the students will like to complete a graduate degree after finishing their undergraduate studies, not necessarily obtaining the certified public accountant certification (CPA). In addition, the principal factors that attracts students to select public accounting as a career are: opportunity to work with different clients and obtain better experience and incentives for professional certifications. The results can be of interest for recruiters in accountant firms because they provide additional information about the needs and perceptions of their prospective future employees. Finally, the results can be applied to other business areas.
\end{abstract}

KEYWORDS: Public accounting, Generation Y, professional career, perceptions 
uando una empresa decide reclutar a un recién graduado de universidad, es probable que seleccione a un miembro de la llamada Generación Y. Esta generación, también conocida como los milenios, son los individuos que nacieron entre 1981 y 1999. De acuerdo con la Oficina de Estadísticas Laborales del Departamento del Trabajo de Estados Unidos (2004), para el 2010 se esperaba que el 60 por ciento de la fuerza trabajadora a tiempo completo en los Estados Unidos fueran trabajadores menores de 34 años. Esta estadística ilustra una posible brecha generacional (entre la Generación $\mathrm{Y}$ y $\mathrm{su}$ antecesora, la Generación X) en el mundo laboral actual (Westerman y Yamamura, 2007). Específicamente, Telberg (citado por Westerman y Yamamura, 2007) reporta que en el área de la contabilidad pública, el 86 por ciento de los Contadores Públicos Autorizados (CPAs) perciben esta brecha generacional en su profesión. Otro estudio de profesionales en finanzas, de la Generación Y, reporta que el 73 por ciento de sus encuestados trabajan con alguien de otra generación (Association of Chartered Certified Accountants, 2010). El hecho de que cada generación posee características particulares y diferentes a las generaciones anteriores representa un posible conflicto en el ambiente de trabajo de una organización.

De acuerdo con Martin (2005), los milenios son personas independientes, con pensamiento empresarial, que demandan retroalimentación inmediata y odian que los estén supervisando constantemente. La expectativa de largo plazo no va más allá de un año.

De acuerdo con una encuesta realizada por la American Institute of Certified Public Accountants (AICPA) (2013), uno de los problemas que tienen las firmas de CPAs comúnmente es el reclutamiento y la retención de empleados. Estas limitaciones de personal han estado entre las primeras cinco dificultades de las firmas de CPAs desde 1999. Una de las posibles razones para explicar la reducción en el interés de proseguir una carrera en contabilidad pública pudiera ser las características y prioridades de los miembros de la Generación Y.

La organización global de contadores públicos $\left(\mathrm{ACCA}^{1}\right.$, por su sigla en inglés) encuestó a sobre 3,200 profesionales de la Generación Y de finanzas de 122 países. El estudio buscaba entender y delinear cómo las aspiraciones y características de estos profesionales moldean lo que los patronos necesitan hacer para tener éxito en atraer, desarrollar y retener a los milenios. El estudio encontró que estos profesionales buscan progresar rápidamente en sus carreras y que son atraídos a un patrono, principalmente, por las alternativas de desarrollo profesional.

Otro resultado del estudio es que los profesionales de la Generación $\mathrm{Y}$ desean una carrera interesante y apasionante, ya sea dentro o fuera del área de finanzas. La ruta profesional que buscan es ambiciosa, flexible, y en evolución constante. Por lo tanto, es imperativo para las organizaciones asegurarse de entender los diferentes carriles profesionales disponibles y comunicarlos claramente a sus empleados prospectos.

Por otro lado, el dinero es importante para esta generación, por lo tanto la remuneración debe ser competitiva y orientada dentro la proposición de trabajo particular. La seguridad laboral también es importante. De este modo, los patronos deben demostrar un ambiente de reclutamiento estable y que sea a largo plazo. Por último, dentro de los factores para atraer a los profesionales de esta Generación, es importante para las organizaciones conocer que los factores de estilo de vida tienen un mayor peso que los factores contractuales.

Dadas estas características de la Generación Y, nos dimos a la tarea de encuestar a estudiantes subgraduados para conocer su sentir sobre el trabajo en la contabilidad pública. Actualmente, en Puerto Rico las firmas de contabilidad pública no

\footnotetext{
${ }^{1}$ Association of Chartered Certified Accountants
} 
están exentas de las limitaciones para reclutar su personal. Estas firmas enfrentan dificultades para contratar estudiantes talentosos inmediatamente después que terminan su bachillerato con especialidad en Contabilidad. Específicamente las grandes firmas de CPAs (Big 4) ${ }^{2}$ enfrentan una competencia proveniente de reclutadores de otras empresas locales y, con una creciente tendencia, de empresas fuera de la isla. No sólo los estudiantes graduados de Contabilidad tienen varias alternativas para elegir a su futuro patrono, sino que existe la realidad de que muchos buscan continuar estudios graduados.

Las firmas de contabilidad pública de Puerto Rico continúan realizando diferentes iniciativas para atraer a los graduandos de Contabilidad. Dentro de estos esfuerzos se encuentran auspiciar programas de orientación a estudiantes talentosos de escuela superior y auspiciar eventos y viajes para estudiantes actuales de la concentración en las diferentes universidades, entre otras actividades. Sin embargo, según señalamientos de los mismos reclutadores de estas firmas, estos esfuerzos todavía no son suficientes. Nuestro estudio consta de una muestra de 172 estudiantes de tercer y cuarto año de la especialidad de contabilidad de una institución de educación superior pública de Puerto Rico. En su mayoría los encuestados son féminas $(67 \%)$, solteros $(90 \%)^{3}$, y entre las edades de 19 a 21 años (57\%). Los resultados de la investigación se presentan en las diferentes tablas que se presentan a continuación (Tablas 1 a la 6).

En las Tablas 1 y 2 se muestran las aspiraciones académicas de los encuestados. Según

\footnotetext{
${ }^{2}$ Las cuatro grandes firmas de contadores y auditores (Big 4) son: KPMG, Ernst \&Young (EY), Deloitte Touche Tohmatsu Limited (Deloitte) y PricewaterhouseCoopers (PwC). Estas cuatro firmas son las mayores redes internacionales de proveedores de servicios profesionales en contabilidad y auditoría, impuestos y consultoría. En mercados fuera de los Estados Unidos también proveen servicios de apoyo corporativo tales como auditoría interna, finanzas y servicios legales.

${ }^{3}$ En este estudio no se consideró la posible existencia de parejas de hecho, esto es, de personas que conviven como pareja sin estar casados entre sí. Esta situación implicaría estudiantes "solteros" con responsabilidades similares a las de una persona casada.
}

muestra la Tabla 1, las aspiraciones académicas de este grupo de estudiantes son muy altas, sólo el 8 por ciento de ellos desea quedarse con el grado de bachillerato. Los grados de maestría y derecho obtuvieron el 35.5 por ciento y 31.4 por ciento, respectivamente.

Es interesante notar que las aspiraciones

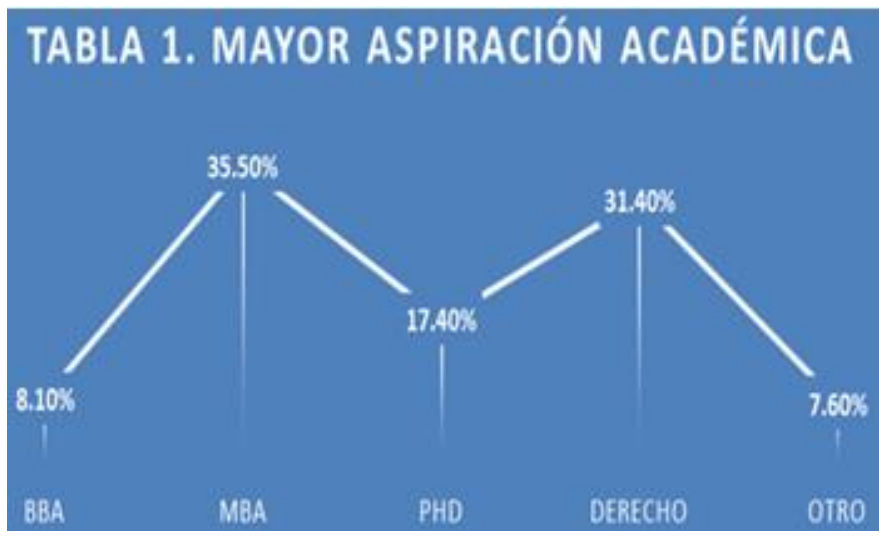

académicas varían por género, según la Tabla 2. En su mayoría las mujeres aspiran a lograr grados mayores que el bachillerato en comparación con los hombres. Este resultado no es sorprendente dada la realidad actual de la educación superior en la que la mayoría de los estudiantes son mujeres.

\section{TABLA 2. MAYOR ASPIRACIÓN ACADÉMICA POR GÉNERO - Hombres aujeres}
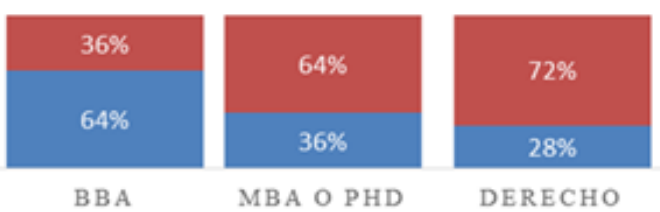

DERECHO

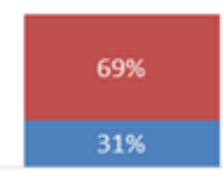

OTRO

Según datos del Consejo de Educación de Puerto Rico, para el 2009-2010, el 59.1 por ciento del estudiantado a nivel superior eran féminas (Calderón Soto, 2012). Otro aspecto a considerar es que actualmente los profesores de Contabilidad poseen estudios post-graduados y alientan a sus estudiantes a proseguir los mismos. 
En cuanto a las certificaciones profesionales, el 100 por ciento de la muestra desea alguna certificación. Sin embargo, es interesante notar que la certificación con mayor popularidad es la de "Certified Management Accountant" (CMA) que no es la certificación profesional del área de contabilidad pública. Si consideramos la totalidad de estudiantes que marcaron la certificación de CMA de alguna manera $\mathrm{u}$ otra (sola o en combinación con alguna otra), el 77 por ciento de la muestra pretende obtenerla. La certificación de Contador Público Autorizado (CPA) por sí sola no fue seleccionada por ninguno de los encuestados. Dicha certificación fue seleccionada por el 50 por ciento de la muestra en conjunto con alguna otra, según muestra la Tabla 3.

\section{TABLA 3. CERTIFICACIONES PROFESIONALES}

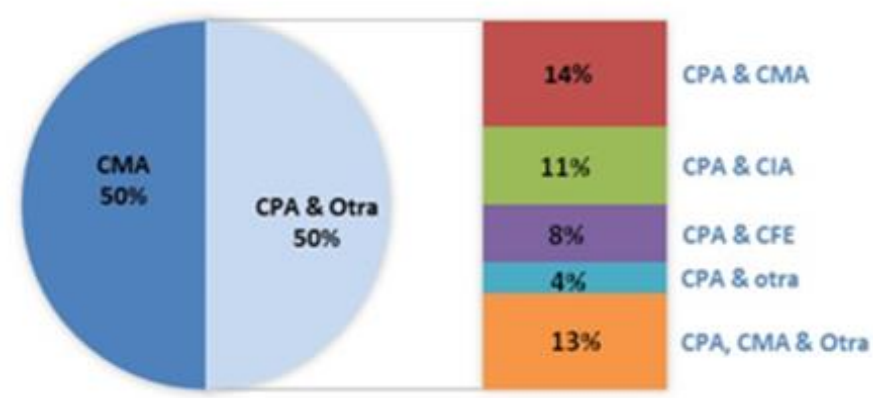

Los resultados muestran la proliferación de certificaciones profesionales en las diferentes áreas de contabilidad. Las certificaciones más populares luego de CMA y CPA fueron "Certified Internal Auditor" (CIA) y "Certified Fraud Examiner" (CFE). Una de las razones para el conocimiento sobre estas certificaciones pudiese ser el hecho de que los estudiantes de Contabilidad de la institución seleccionada deben tomar auditoría como requisito de su grado. Por lo tanto, dichas certificaciones se discuten y son conocidas por los estudiantes.

Estos resultados contrastan con las aspiraciones de la generación anterior a esta. A base de la experiencia anecdótica obtenida de otros miembros de la Generación X (incluyendo a la autora), aprobar el examen de reválida para obtener el título o certificación profesional de CPA era el siguiente logro necesario en la carrera profesional de un egresado de universidad con un grado en Contabilidad. Una posible explicación para esto es que los estudiantes de Contabilidad ahora tienen varias alternativas a cuanto a certificaciones profesionales que antes no existían. Otra posible explicación pudiese ser que los estudiantes no saben qué es ser CPA o lo que conlleva esta carrera, que carecen de un modelo cercano a quien emular. Se encontró que el 71 por ciento de los encuestados no tienen un familiar cercano que fuera CPA. Más aún, es la percepción de la autora, que en estos tiempos los profesores de Contabilidad cada vez más se dedican a la cátedra a tiempo completo y no mantienen una práctica privada como en el pasado ${ }^{4}$.

Para conocer las percepciones ${ }^{5}$ de los estudiantes sobre la contabilidad pública a los encuestados se les entregó una lista de aseveraciones con características o factores del trabajo para que opinaran sobre estas. Estas características del trabajo incluían por ejemplo: "La contabilidad pública paga los salarios iniciales más altos" y "En la contabilidad pública existe mayor oportunidad de ascensos". Cada una de las oraciones se evaluaba en una escala tipo Likert de siete puntos que incluía las siguientes categorías: completamente de acuerdo, en acuerdo, parcialmente de acuerdo, neutral, parcialmente en desacuerdo, en desacuerdo y completamente en desacuerdo. Las categorías se valoraron del uno al siete para obtener los resultados estadísticos. La categoría con mayor valor (7) es completamente de acuerdo y con menor valor (1) es completamente en desacuerdo. En términos de interpretación a mayor el promedio obtenido por la característica, mejor es la percepción de estudiante hacia la

\footnotetext{
${ }^{4}$ En la institución universitaria donde la autora trabaja, la mayoría de los profesores de Contabilidad están dedicados a llevar a cabo tareas de investigación y enseñanza en el salón de clase a tiempo completo. Es posible que en otras instituciones universitarias la realidad sea diferente.

${ }^{5}$ Las percepciones es lo que piensan u opinan los estudiantes sobre la contabilidad pública.
} 
misma. En otras palabras, más en acuerdo el estudiante está con la misma.

\section{TABLA 4. PRINCIPALES FACTORES DE LA CONTABILIDAD PÚBLICA:}

\section{En la contabilidad pública existe una mayor oportunidad de trabajar:}

\begin{tabular}{|c|c|}
\hline - con diferentes clientes. & 5.61 \\
\hline - para obtener mejor experiencia e incentivos & 5.44 \\
\hline - para recibir educación continua. & 5.31 \\
\hline $\begin{array}{l}\text { - con otros profesionales (colegas y } \\
\text { superiores). }\end{array}$ & 5.21 \\
\hline - horas extras. & 5.11 \\
\hline - por un largo periodo de tiempo. & 5.11 \\
\hline
\end{tabular}

Las mujeres asignaron el segundo lugar en importancia al hecho que las firmas de contabilidad pública proveen una mejor experiencia e incentivos para obtener certificaciones profesionales. Sin embargo, esta característica los hombres la situaron en un tercer lugar. Las estudiantes perciben que en la contabilidad pública existe una mayor oportunidad de recibir educación continua en un tercer lugar y los estudiantes en un quinto lugar. Por otro lado, las féminas posicionaron el tener una mayor oportunidad de trabajar con otros profesionales como colegas y superiores en un cuarto lugar, mientras que los hombres la posicionaron en un segundo lugar. Las damas perciben en quinto lugar que se van a trabajar una cantidad mayor de horas extras en la contabilidad pública.

\section{TABLA 5. PERCEPCIONES DEL TRABAJO EN LA CONTABILIDAD PÚBLICA POR GÉNERO:}

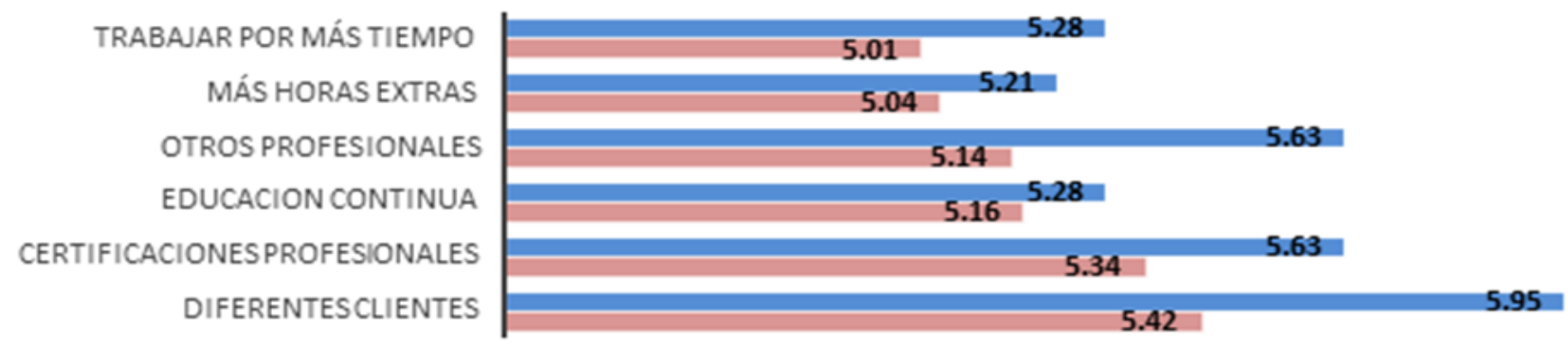

- Hombre = Mujer

Las Tablas 4 y 5 incluyen las seis características que obtuvieron los promedios mayores. En la Tabla 4 se reportan los factores al considerar ambos géneros y en la Tabla 5 se presentan los promedios de las respuestas por género. En términos generales, la oportunidad de trabajar con diferentes clientes fue la característica que recibió un mayor promedio para ambos géneros (5.61). Para el resto de los factores el orden varía por género ${ }^{6}$.

${ }^{6}$ Se hicieron pruebas T (Student t-test, por su nombre en inglés) para determinar si las diferencias en promedios por género para los factores eran estadísticamente válidas.
Sin embargo, esta característica obtuvo el sexto lugar entre los caballeros. Por último, en cuarto lugar los hombres perciben que en la contabilidad pública existe una mayor oportunidad de trabajar por un largo tiempo, característica que ocupa el sexto lugar entre las mujeres.

Uno de los rasgos de la Generación Y es la importancia que le da a la remuneración (ACCA, 2010). Como parte del estudio se incluyeron

Permitiendo un nivel de error del cinco por ciento, dos de los factores fueron significativos: diferentes clientes y otros profesionales. 
aseveraciones relacionadas al salario, bonos y beneficios marginales. Aunque estas características no son percibidas dentro de las seis más importantes, sí se posicionaron dentro del segundo grupo de importancia. En la Tabla 6, se presentan los resultados para las aseveraciones relacionadas con la remuneración. Se presenta el promedio total $\mathrm{y}$ el porciento de estudiantes por género que contestaron completamente de acuerdo, en acuerdo o parcialmente en acuerdo. Se puede decir que los estudiantes en general perciben lo relacionado a salario, bonos y beneficios marginales en la contabilidad pública como neutral (promedios totales van de 4.84 a 4.03). En su mayoría los hombres tienen una percepción mejor sobre estos temas que las mujeres.

TABLA 6. PERCEPCIONES SOBRE LA REMUNERACIÓN Y BENEFICIOS EN LA CONTABILIDAD PÚBLICA

\begin{tabular}{|c|c|c|c|}
\hline $\begin{array}{l}\text { La contabilidad pública } \\
\text { paga/provee: }\end{array}$ & $\begin{array}{l}\text { Promed } \\
\text { io }\end{array}$ & $\begin{array}{l}\text { Hombre } \\
\text { s }\end{array}$ & $\begin{array}{l}\text { Mujere } \\
\text { s }\end{array}$ \\
\hline - salarios más altos a largo plazo. & 4.84 & $61 \%$ & $60 \%$ \\
\hline - salarios iniciales más altos. & 4.6 & $54 \%$ & $55 \%$ \\
\hline - mejores planes de bonificación. & 4.57 & $46 \%$ & $46 \%$ \\
\hline $\begin{array}{l}\text { - mejores beneficios de licencia de } \\
\text { maternidad/paternidad. }\end{array}$ & 4.56 & $47 \%$ & $47 \%$ \\
\hline - mejores beneficios médicos. & 4.51 & $48 \%$ & $43 \%$ \\
\hline - mejor las horas extras. & 4.41 & $48 \%$ & $50 \%$ \\
\hline - mejores planes de retiro. & 4.3 & $37 \%$ & $35 \%$ \\
\hline -bonos de entrada más altos. & 4.29 & $39 \%$ & $29 \%$ \\
\hline -más tiempo para vacaciones. & 4.04 & $34 \%$ & $31 \%$ \\
\hline $\begin{array}{l}\text { - mayor flexibilidad para obtener las } \\
\text { horas extras trabajadas como } \\
\text { vacaciones. }\end{array}$ & 4.03 & $26 \%$ & $25 \%$ \\
\hline \multicolumn{4}{|c|}{$\begin{array}{l}\text { Nota: El promedio representa el promedio total de ambos géneros. Las } \\
\text { columnas de hombres y mujeres reportan los porcientos de los participantes } \\
\text { que marcaron parcialmente de acuerdo, en acuerdo y completamente de } \\
\text { acuerdo. }\end{array}$} \\
\hline
\end{tabular}

Como parte de esta investigación se llevaron a cabo entrevistas a personal de reclutamiento de las cuatro grandes firmas de contabilidad pública en Puerto Rico. Durante estas entrevistas, los reclutadores nos indicaron que sus firmas promovían el balance entre el trabajo y la vida personal al igual que el trabajo comunitario entre sus prospectos porque estos aspectos eran importantes para la Generación Y. Sin embargo, nuestros resultados indican lo contrario. La percepción sobre un mejor balance entre el trabajo y la vida personal obtuvo solo un 3.87 de promedio. Por otro lado, el que la contabilidad pública provee mayores oportunidades para hacer trabajo comunitario obtuvo un 4.23.

Estos promedios indican que la percepción hacia la premisa sobre "balance entre el trabajo y la vida personal", así como "oportunidades para realizar trabajo comunitario" no son consistentes con los esfuerzos que llevan a cabo las firmas para comunicar a los candidatos la disponibilidad de estas oportunidades, toda vez que el promedio de 4 proyecta neutralidad. Además están parcialmente en desacuerdo con que se proveen mayores oportunidades para hacer trabajo comunitario.

En relación con la urgencia de retroalimentación inmediata en la Generación Y, argumentada en otros estudios, nuestros resultados la avalan. Específicamente, encontramos que los estudiantes le dieron un promedio mayor de cinco a todo lo relacionado con retroalimentación. Este promedio significa que en general, los estudiantes están entre parcialmente de acuerdo o en acuerdo con que existe una mayor retroalimentación cuando trabajan en la contabilidad pública que otras áreas de contabilidad.

Los resultados de esta investigación pueden ser de interés para las personas que llevan a cabo tareas de reclutamiento para las firmas de contabilidad porque aporta información adicional sobre las necesidades y percepciones de sus futuros empleados. También pueden ser aplicados a otras áreas de los negocios. A continuación se ofrecen recomendaciones a la hora de reclutar a prospectos de la Generación Y, específicamente en el área de contabilidad pública:

1. Se debe enfatizar en la posibilidad de trabajar con diferentes clientes a través de la carrera en contabilidad pública. Este énfasis se debe incluir desde las orientaciones que se le dan a los estudiantes de escuela superior ya que pudiese servir de 
inspiración a futuros estudiantes de contabilidad.

2. Se debe proporcionar oportunidades de desarrollo profesional, sea a través de certificaciones profesionales, educación continua o estudios graduados. Estas oportunidades deben dejárseles saber a los prospectos desde temprano en el proceso de reclutamiento, ya que este factor tiene mucho valor para los milenios. Recordemos que solo el ocho por ciento piensa el bachillerato como aspiración académica mayor.

3. Dada la percepción de que se va a trabajar más horas extras en la contabilidad pública, las firmas deben brindar espacio y tiempo a los candidatos a las diferentes certificaciones profesionales o estudios graduados. De existir estos beneficios se deben promocionar entre los prospectos de empleo. Se debe proporcionar suficiente flexibilidad para acomodar las diferentes exigencias que estos empleados pudiesen tener.

4. Los salarios, bonos y salarios son importantes para la Generación Y, pero estos no se perciben como altos o mayores en la contabilidad pública entre los estudiantes. Las firmas de contabilidad pública deben promulgar su esquema de remuneración de manera más efectiva para que logren atraer a más candidatos.

5. Aunque existen esfuerzos de parte de las firmas de contabilidad para crear un mejor balance entre la vida y el trabajo de sus empleados, este factor no es reconocido por los estudiantes de contabilidad. Las firmas deben comunicar mejor las alternativas de flexibilidad en la jornada de trabajo que existen para que los estudiantes las entiendan.

\section{Referencias:}

American Institute of Certified Public Accountants (2013). 2013 PCPS CPA Firm Top Issues, AICPA, disponible en:

http://www.aicpa.org/InterestAreas/PrivateCompaniesPra cticeSection/StrategyPlanning/FirmStrategyandPlanning/p ages/pcps\%20top\%20issues\%20survey.aspx.

Association of Chartered Certified Accountants (2010). Generation Y: Realizing the potential, London, UK: Lyon, J., Filmer, S., \& McDougall, B.

Calderón Soto, J. (2012). Perfil del estudiantado universitario en Puerto Rico: Hallazgos del Consejo de Educación. Disponible en http://www.ce.pr.gov/.

Martin, C. A. (2005). From high maintenance to high productivity. What managers need to know about Generation Y. Industrial and Commercial Training, $37(1), 39-44$.

Oficina de Estadisticas Laborales (2004). Occupational Outlook Quarterly, Invierno 2003 - 2004, p.46 disponible en: http://bls.gov/opub/ooq/2003/winter/art05.pdf

Westerman, J. W. \& Yamamura, J. H. (2007). Generational preferences for work environment fit: effects on employee outcomes. Career Development International, 12(2), $150-161$.

\section{Información de la autora}

Marisela Santiago Castro

prof.msantiagocastro@gmail.com

Catedrática Asociada de la Facultad de Administración de Empresas y la Directora Asociada del Programa de Estudios de Honor de la Universidad de Puerto Rico, Recinto de Río Piedras. Sus intereses de investigación son gobierno corporativo, emprendimiento y educación contable. 